\section{Transform techniques in chemistry}

Transform Techniques in Chemistry. Edited by P. R. Griffiths. Pp. 385. (Plenum: New York and London, 1978.) $\$ 47.40$.

MaNY spectroscopists, particularly those using nuclear magnetic resonance, have had their field revolutionised in the past few years by the introduction of Fourier transform techniques, but have been forced to pick up the new folklore in a haphazard manner for lack of a suitable general treatment of transform methods written from the point of view of the chemist. This excellent new book goes a long way towards satisfying this need. It provides a convincing demonstration that the concept of Fourier transformation is common to a whole range of physical techniques, ranging from the well-known NMR spectroscopy and infra-red interferometry to the more recent Fourier transform ion cyclotron resonance. Although Fourier transformation naturally dominates the discussion, it is shown that related techniques such as the Hadamard and Walsh transforms can also be useful to the spectroscopist in suitable circumstances.

This book is a collection of chapters by different authors, and can be divided into the mathematical and data handling topics (chapters 2-4, 7, and 11-13) and applications to specific types of spectroscopy or chemistry (chapters 1, $5,6,8-10$ and 14). This method of compiling a book has the obvious strength of allowing the important sections to be written by the appropriate expert; in the present case there is an excellent description of Fourier transform ion cyclotron resonance by Comisarow. On the other hand, multiple authorship can mean undesirable repetition. Worse still, a given topic may be treated superficially several times over, no single author wishing to devote enough space for a definitive discussion. For example, the practice of 'zero-filling' interferograms raises some fairly fundamental questions that are seldom answered to the satisfaction of the average spectroscopist, and one would have expected the present volume to tackle this hot potato once and for all. Instead the subject is treated separately in chapters 4,8 and 11 , in each case rather uncritically.

This is an extremely useful book for locating those elusive but vital pieces of information the research worker always seems to be looking for; at the same time the general concepts are very well treated. Most readers will be surprised at the number of different areas where transform techniques are used and how related problems turn up in different forms in the different fields. This broadening of our understanding could well lead to new experiments inspired by the methods used by our colleagues. Everyone will find something new in this book. Even the jargon of our fellow spectroscopists can come as a surprise; the reviewer particularly noticed "dichotomizer", "crosscorrelogram", "decimation" and "transgeneration".

In spite of the use of multiple authors, the material presented is remarkably up-to-date, and there seem to be no glaring omissions. It would have been nice, however, to have seen some mention of Fourier transform methods of NMR imaging (zeugmatography) and the developing field of two-dimensional Fourier transformation.

Ray Freeman

Ray Freeman is Lecturer in the Physical Chemistry Laboratory at the University of Oxford, UK.

\section{Soil profile recording}

Soil Sampling and Soil Description. By J. M. Hodgson. Pp.241. (Clarendon Oxford University Press: Oxford. 1978.) £10.

FrOM the outset it is essential to realise that this little book is not intended to be a field handbook. Rather it is a review of 'how and why' specific profile features should be recorded together with a summary of the descriptive methods adopted by various national and international organisations

In the opening chapter the author considers the reasons for studying the soil in the field and the need to describe accurately profile features. Chapters 2 and 3 provide a concise review of methods of profile site selection, description and preparation.

The 'how and why' of soil description is the central theme of chapter 4 , which provides a comprehensive list of the soil features the field scientist is likely to encounter. Unfortunately the approach adopted in this chapter is by no means uniform. Each feature is dealt with in turn; in some instances the reasons for selecting a particular feature are discussed in detail, whereas in others the importance is assumed to be obvious. Similarly when dealing with possible means of measuring or classifying a particular feature the methods are sometimes considered in detail. as for example in the case of structure or as in the case of roots only perfunctorily.

This long account is followed by two succinct chapters covering techniques of data recording and sample collection. Of particular value is the section on recording of data in a form amenable to computer storage. (In this section figures 5.5 and 5.8 are transposed.) The final chapter is in the form of a summary of the profile features used in soil description by some 28 different organisations. The reviews are necessarily brief and details are only given where the procedure or feature used differs from those in the USDA 1951 Handbook. This chapter is valuable if only because it brings together for the first time the approaches adopted by a variety of agencies.

Overall one is left with the question of what is the purpose of this book? Had the chapters on the 'how and why' of soil description considered the question of "why" in greater depth then this would indeed have been a useful text.

M. J. Alexander

M. J. Alexander is Lecturer in Geography at the University of Durham, UK.

\section{SCIENTIFIC - BOOKS}

H. K. LEWIS can supply works in all branches of Pure and Applied Science. Catalogues on request. Please state interests.

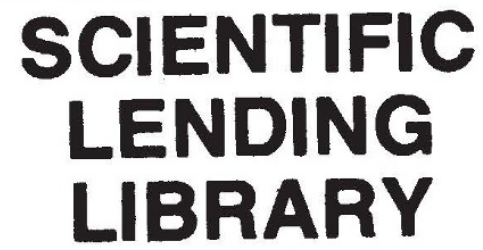

Annual Subecription from \$7.00. (Available in U.K. only)

Reduced rates for multiple subscriptions.

Prospectus post free on request.

Quarterly List of Now Books and now editions added to the Library sent post free to subscribers regularly.

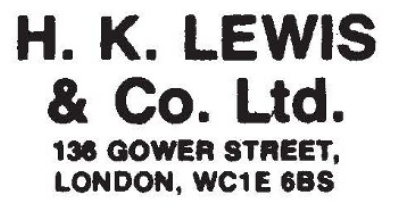

Telophone: 01-307 4242

Teloreme: "Publicenl",

London, wCiE Ges."

Circle No. 10 on Reader Enquiry Card. 